\title{
Parallel Strong-Strong/Strong-Weak Simulations of Beam-Beam Interaction in Hadron Accelerators
}

\author{
Ji Qiang*, Miguel Furman*, Robert D. Ryne*, Wolfram Fischer ${ }^{\dagger}$, Tanaji Sen** and \\ Meiqin Xiao** \\ *Lawrence Berkeley National Laboratory, Berkeley, CA 94720 \\ ${ }^{\dagger}$ Brookhaven National Laboratory, Upton, NY 11973 \\ ${ }^{* *}$ Fermi National Laboratory, Batavia, IL 60510
}

\begin{abstract}
In this paper, we present the results of using a parallel computational tool, BeamBeam3D, developed at Lawrence Berkeley National Laboratory, for strong-strong/strong-weak modeling of the beam-beam effects in three hadron accelerators: RHIC, Tevatron and LHC. This tool calculates self-consistently the electromagnetic beam-beam forces for arbitrary distributions and separation during each collision when a strong-strong beam-beam interaction model is used. When a strong-weak model is used, the code has the option of using a Gaussian approximation for the strong beam. Using the strong-strong model, we have studied the effect of time modulated offset beam-beam interaction on the emittance growth in the RHIC and LHC. We observed an extra $0.04 \%$ emittance growth after 300,000 turns in the RHIC where the time-averaged beam-beam offset is one transverse rms beam size and the modulation frequency is $10 \mathrm{~Hz}$. There is no significant additional emittance growth in the LHC after one million turns where the time-averaged offset is zero. Using the strong-weak model, we have also studied the antiproton lifetime subject to 72 long range beam-beam interactions at $150 \mathrm{GeV}$ injection energy in the Tevatron. The simulation shows a qualitative agreement with the experimental observation of the smaller antiproton emittance having a longer lifetime.
\end{abstract}

\section{INTRODUCTION}

The beam-beam interaction puts a strong limit on the luminosity of the high energy hadron colliders. For example, in the Tevatron Run II experiment, long-range beambeam forces significantly reduce the antiproton lifetime and is one of the major factors preventing the achievement of designed luminosity. To study the beam-beam interaction, an important approach is to use self-consistent macroparticle simulation. However, in the hadron accelerators, radiation damping is very weak for hadron particles. It requires to track the particles for many thousand turns to study the long-term emittance growth and particle losses. Using a parallel beam-beam computational tool will significantly reduce the study time. Meanwhile, it also provides the capability to include more complex physical process and to do the simulations with higher numerical accuracy.

\section{COMPUTATIONAL MODEL}

In the computational beam-beam model, we have used six dimensional phase space coordinates $\left(x, p_{x}, y, p_{y}, \Delta z, \Delta p_{z} / p_{0}\right)$ to describe the particle motion in the accelerator. Here, $p_{x, y}$ is the transverse momentum normalized by the total momentum of a reference particle $\left(p_{0}=E_{0} / c\right), \Delta z=s-c t(s)$ with $c$ the speed of light, and $\Delta p_{z}=|p|-p_{0}$. To calculate the electromagnetic force from the beam-beam interaction, we have used a multiple slice model. In this model, each beam bunch is divided into a number of slices along the longitudinal direction in the moving frame. Each slice contains nearly the same number of particles at different longitudinal locations $\Delta z$. The collision point between two opposite slices $i$ and $j$ is determined by

$$
s_{c}=\frac{1}{2}\left(\Delta z_{i}^{+}-\Delta z_{j}^{-}\right)
$$

The transverse coordinates of the particles at the collision point are given by

$$
\begin{aligned}
& x^{c}=x+s_{c} p_{x} \\
& y^{c}=y+s_{c} p_{y}
\end{aligned}
$$

The slopes of the particles are updated using the beambeam electromagnetic forces at the collision point following

$$
\begin{aligned}
& p_{x_{\text {new }}}=p_{x}+\Delta p_{x} \\
& p_{y_{\text {new }}}=p_{y}+\Delta p_{y}
\end{aligned}
$$


where

$$
\begin{aligned}
\Delta p_{x_{2}} & =\frac{2 q_{1} q_{2} N_{1}}{\gamma_{2} 4 \pi \varepsilon_{0} m_{2} c^{2}} E_{x_{1}} \\
\Delta p_{y_{2}} & =\frac{2 q_{1} q_{2} N_{1}}{\gamma_{2} 4 \pi \varepsilon_{0} m_{2} c^{2}} E_{y_{1}} .
\end{aligned}
$$

In the above equations, the subscripts 1 and 2 pertain to each of the two beams, the corresponding equations for the other beam are obtained from the above by the exchange $1<->2, \gamma=1 / \sqrt{1-\beta^{2}}, \beta_{i}=v_{i} / c, i=x, y, z, c$ is the speed of light, $\varepsilon_{0}$ is the vacuum permittivity, $q$ is the charge of the particle, $m$ is the rest mass of particle, $N$ is the number of particles in a bunch, and $E_{x}$ and $E_{y}$ are the transverse electric fields generated by the opposite moving beam. After the collision, the particles of each slice drift back to their original locations according to

$$
\begin{aligned}
& x=x^{c}-s_{c} p_{x_{\text {new }}} \\
& y=y^{c}-s_{c} p_{y_{\text {new }}}
\end{aligned}
$$

The electric fields generated by the opposite moving beam can be obtained from the solution of Poisson's equation. The solution of Poisson's equation can be written as

$$
\phi(x, y)=\int G(x, \bar{x}, y, \bar{y}) \rho(\bar{x}, \bar{y}) d \bar{x} d \bar{y}
$$

where $G$ is the Green's function and $\rho$ is the charge density. For the case of transverse open boundary conditions, the Green's function is given by:

$$
G(x, \bar{x}, y, \bar{y})=-\frac{1}{2} \ln \left((x-\bar{x})^{2}+(y-\bar{y})^{2}\right)
$$

The convolution for $\phi$ in Eq. 10 can be computed efficiently using an FFT in the doubled computational domain as described by Hockney and Eastwood. [1].

In the FFT-based algorithm, the particle domain and the electric field domain are contained in the same computational domain. Here, the particle domain is the configuration space containing the charged particles, and the field domain is the space where the electric field is generated by the charged particles. In the beam-beam interaction, the two opposite moving beams might not overlap with each other. For example, in the long-range interaction, the two colliding beams could be separated by more than several $\sigma$, where $\sigma$ is the rms size of the beam. Thus the field domain where the electric field is generated by one beam can be different from the particle domain containing the beam. In the beam-beam simulation, the origin of the field domain can be at an arbitrary location and varies from turn to turn. To apply Hockney's algorithm directly will require the computational domain to contain both the particle domain and the field domain, i.e. both beams. Since there is a large empty space between two beams, containing both beams in one computational domain will result in a poor spatial resolution of the beams. This is also computationally inefficient because the electric fields in the empty space between two beams are not used.

To avoid this problem, we have defined a shifted Green function as

$$
G_{s}(x, \bar{x}, y, \bar{y})=-\frac{1}{2} \ln \left(\left(x_{c}+x-\bar{x}\right)^{2}+\left(y_{c}+y-\bar{y}\right)^{2}\right)
$$

where $x_{c}$ and $y_{c}$ are the center coordinates of the field domain. The electric potential in the field domain is written as

$$
\phi\left(x+x_{c}, y+y_{c}\right)=\int G_{s}(x, \bar{x}, y, \bar{y}) \rho(\bar{x}, \bar{y}) d \bar{x} d \bar{y} .
$$

Using the shifted Green function, the center of the field domain is shifted to the center of the particle domain. The range of $x$ and $y$ cover both the particle domain and the field domain in one computational domain. The FFT can be used to calculate the convolution in Eq. 10 using the new Green function. A more detailed discussion of the shifted Green function method can be found in [2].

To summarize, using the shifted Green function:

- avoids the requirement that the particle domain and the field domain be contained in one big computational domain,

- leads to better numerical resolution for the charge densities and the resulting electric fields than the conventional method, because the empty space between the beams is not included in the calculation,

- is far more efficient, in terms of computational effort and storage, than the traditional approach of gridding the entire problem domain.

When the strong-weak beam-beam model is used, the program has the option to use the electric field from the numerical solution of Poisson's equation or from the Gaussian approximation of the strong beam. In the latter case, the electric field is calculated following a Gaussian code [3].

The effects of external fields can be represented, in the small-amplitude approximation, by a linear transfer map between collision points. A one-turn map is included to take into account the linear machine chromaticity effect. The effects of radiation damping and quantum excitation are represented using a localized stochastic map.

\section{STRONG-STRONG SIMULATIONS OF BEAM-BEAM INTERACTION IN RHIC AND LHC}

In the hadron colliders, the closed orbit of each bunch at interaction point can be perturbed due to the vibration of focusing magnets or due to parasitic beam-beam 
TABLE 1. RHIC Physical Parameters for the Beam-Beam Simulations

\begin{tabular}{ll}
\hline beam energy $(\mathrm{GeV})$ & 23.4 \\
protons per bunch & $8.4 \times 10^{10}$ \\
$\boldsymbol{\beta}^{*}(\mathrm{~m})$ & 3.0 \\
RMS spot size at the IP $(\mathrm{mm})$ & 0.629 \\
betatron tunes $\left(v_{x}, v_{y}\right)$ & $(0.22,0.23)$ \\
chromaticity $\left(q_{x}^{\prime}, q_{y}^{\prime}\right)$ & $(2,2)$ \\
synchrotron tune $\boldsymbol{v}_{z}$ & $3.7 \mathrm{e}-4$ \\
RMS bunch length $(\mathrm{m})$ & 3.6 \\
momentum spread & $1.6 \mathrm{e}-3$ \\
offsel (sigma) & 1 \\
oscillation frcquency $(\mathrm{Hz})$ & 10 \\
\hline
\end{tabular}

interaction. This results in the bunches colliding at the interaction point with a small transverse offset. For example, in RHIC, there exists triplet vibration with frequency close to $10 \mathrm{~Hz}$, which leads to offset of $5-10 \%$ of one $\sigma$ at the interaction point [4]. For practical purposes, it is important to know if offsets (static and modulated) cause additional emittance growth. To study this effect, we have carried out a strong-strong beam-beam simulation of two proton beams colliding in RHIC. The major physical parameters used in the simulation are given in Table 1. Here, we have chosen an offset of one $\sigma$ from the closed orbit in order to maximize its effect since the beam-beam force is strongest around one $\sigma$ for a Gaussian density distribution. The initial distribution was Gaussian but was allowed to evolve freely. We first studied the proton emittance growth in RHIC with static offset beam-beam collision. Fig. 1 shows a comparison of the emittance evolution (averaged over horizontal and vertical plane) with and without offset beam-beam collision. We see that emittance growth after 300,000 turns

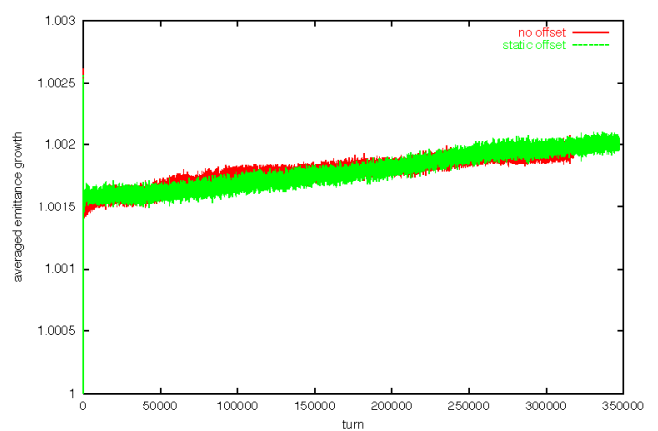

FIGURE 1. Fmittance evolution (averaged over horizontal and vertical plane) with and without a one $\sigma$ offset beam-beam collision in RHIC.

is about the same with and without the offset. This suggests that the static beam-beam offset collision will not cause an extra emittance growth. The averaged emittance has grown by about $0.05 \%$ after 300,000 turns. This emittance growth is driven by the nonlinear electromag-
TABLE 2. LHC nominal beam-beam parame-

\begin{tabular}{ll} 
ters & \\
\hline beam energy $(\mathrm{TeV})$ & 7 \\
protons per bunch & $1.05 \times 10^{11}$ \\
$\beta^{*}(\mathrm{~m})$ & 0.5 \\
RMS spot size at the IP $(\mu m)$ & 15.9 \\
betatron tunes $\left(v_{x}, v_{y}\right)$ & $(0.31,0.32)$ \\
synchrotron tune $v_{z}$ & 0.0021 \\
RMS bunch length $(\mathrm{m})$ & 0.077 \\
momentum spread & $1.11 \mathrm{e}-4$ \\
\hline
\end{tabular}

netic forces during the beam-beam interaction. Next, we studied the emittance growth with time modulated offset beam-beam collision. Here, we have assumed $10 \%$ oscillation amplitude around one $\sigma$ time-averaged offset in the horizontal direction. The oscillation frequency is set as $10 \mathrm{~Hz}$ to emulate the triplet vibration in RHIC. Fig. 2 shows a comparison of the averaged emittance evolution with and without offset beam-beam collision. With the time modulated offset beam-beam collision, the

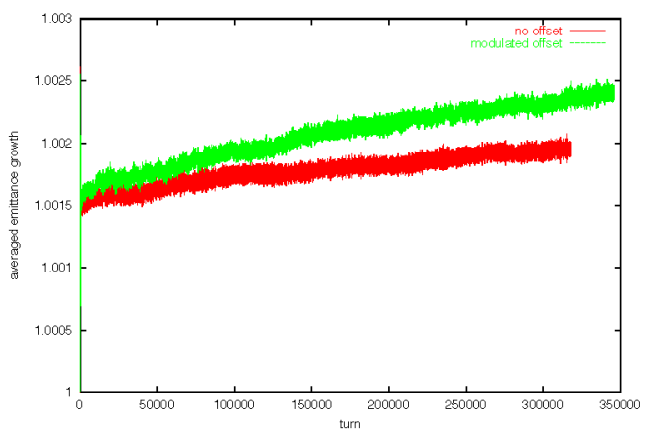

FIGURE 2. Emittance evolution (averaged over horizontal and vertical plane) with and without a time-modulated off set beam-beam collision in RHIC.

averaged emittance growth is about $0.09 \%$ after 300,000 turns. There is about $0.04 \%$ extra emittance growth due to the effect of time modulated beam-beam collision. In this simulation for RHIC, we have assumed that time-

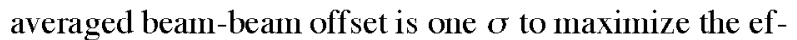
fect from time modulated offset beam-beam collision. In LHC, it is estimated that the time-averaged beam-beam offset is about zero but with about 0.10 bunch-to-bunch closed orbit variation due to the parasitic beam-beam interaction [5]. We have done a strong-strong simulation using a set of nominal LHC physical parameters with $0.1 \sigma$ oscillation amplitude and 1000 turn oscillation period. The nominal beam-beam parameters of the LHC are given in Table 2. Fig. 3 shows the emiltance growth with and without the time modulated beam-beam collision. We see that there is about $0.05 \%$ emittance growth after one million turns with the nominal head-on collision. Even though the final emittance from the time modulated beam-beam collision is slightly higher than that without 


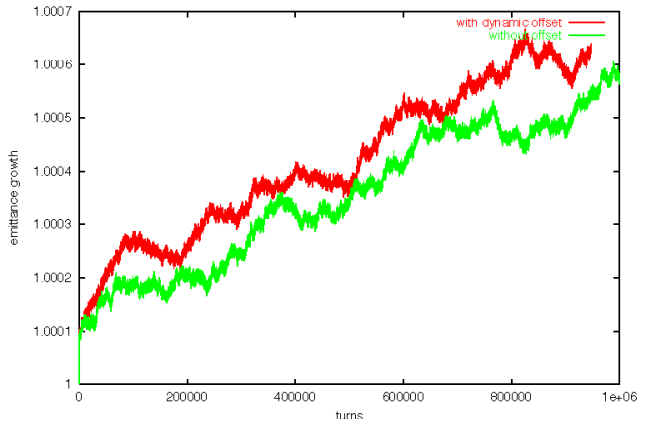

FIGURE 3. Emittance evolution (averaged over horizontal and vertical plane) with and without offset beam-beam collision in $\mathrm{LHC}$.

offset, the emittance growth rate is about the same. All the above simulations have been done using one million particles for each beam, $128 \times 128$ mesh points, and single slice model.

\section{STRONG-WEAK SIMULATION OF LONG RANGE BEAM-BEAM INTERACTION IN TEVATRON}

We have assumed a strong-weak beam-beam interaction model in the calculation of the antiproton lifetime since the antiproton intensity is much smaller than the proton intensity (typically a factor of 10 ). There are 72 long range beam-beam interaction points at the $150 \mathrm{GeV}$ injection stage of the Tevatron. The linear transfer maps between collision points for antiproton particles are obtained from the MAD simulation of the Tevatron lattice. At the collision point, every antiproton receives a kick from the beam-beam force generated by the proton beam. The beam-beam force is calculated assuming a Gaussian distribution for the proton beam. After each turn, a linear chromaticity map and a random diffusion map are applied to all antiprotons. The random kick was added to simulate the effect of gas scattering which has a sizable influence on the emittance growth at injection. The simulations are run for 100,000 turns using one million particles. In the simulation, we have assumed an "aperture" size of $3.25 \sigma$, where the $\sigma$ is the horizontal or vertical rms size at each collision point. The aperture is such that a particle is lost with a unit probability when it hits it. The antiproton lifetime $\tau$ is estimated from fitting the antiprotron intensity with function $I_{0} \exp (-t / \tau)$ using a least square method. Here, $I_{0}$ is the initial antiproton intensity.

Fig. 4 shows the antiproton lifetime as a function of the initial antiproton emittance. With a factor 2 increase of the antiproton emittance, the antiproton lifetime de-

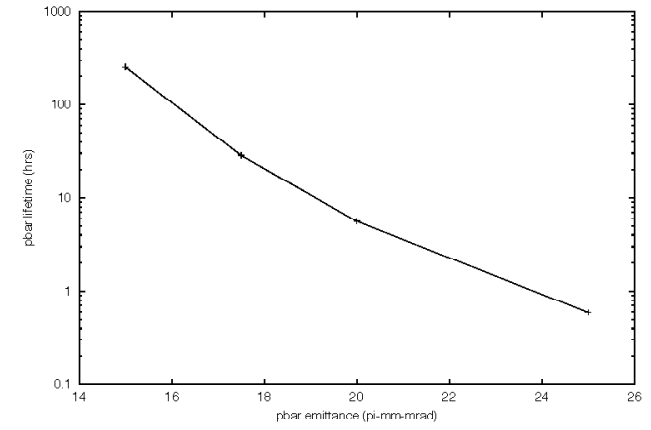

FIGURE 4. Antiproton lifetime as a function of antiproton emittance at $150 \mathrm{GeV}$ Tevatron.

creased drastically by more than a factor of 100 . The strong antiproton emittance dependency of the lifetime may be due to the following two effects: On the one hand, the larger antiproton emittance gives a larger antiproton beam size and results in a faster loss to the aperture. On the other hand, the larger antiproton beam size reduces the distance between the proton beam and the antiproton particles, which results in stronger nonlinear beam-beam interactions.

\section{ACKNOWLEDGMENTS}

This work was performed using NERSC and CCS supercomputers under the auspices of a SciDAC project, "Advanced Computing for 21 st Century Accelerator Science and Technology, which is supported by the US DOE/SC Office of HENP and the Office of ASCR under contract DE-AC03-76SF00098.

\section{REFERENCES}

1. R. W. Hockney and J. E. Eastwood, "Computer Simulation Using Particles," McGraw-Hill Book Company, New York, 1985.

2. J. Qiang, M. A. Furman, R. D. Ryne, Phys. Rev. ST Accel. Beams 5, 104402 (2002).

3. M. A. Furman, "Beam-Beam Simulations with the Gaussian Code TRS," in Proc. ICAP98, Monterey, California, Sept. 14-18, 1998.

4. C. Montag, J. M. Brennan, J. Butler, and P. Koello, "Measurements of mechanical triplet vibrations in RIIIC," Proc. of the 2002 EPAC, Paris, France (2002).

5. W. Herr, "Beam-beam issues in the LHC and relevant experience from the SPS proton antiproton collider and LEP," in Proceedings of a Workshop on Beam-beam Effects in Circular Colliders, Fermilab, June 25-27, 2001. 